\title{
Behavioural biology of Chagas disease vectors
}

\author{
Claudio Ricardo Lazzari¹/+, Marcos Horácio Pereira², Marcelo Gustavo Lorenzo ${ }^{3}$ \\ IInstitut de Recherche sur la Biologie de I'Insecte, Unité Mixte de Recherche 7261, Centre National de la Recherche Scientifique, \\ Université François Rabelais de Tours, Tours, Indre et Loire, France 2 Instituto de Ciências Biológicas, Universidade Federal de Minas Gerais, \\ Belo Horizonte, MG, Brasil ${ }^{3}$ Centro de Pesquisa René Rachou-Fiocruz, Belo Horizonte, MG, Brasil
}

Many arthropod species have adopted vertebrate blood as their main food source. Blood is rich in nutrients and, except for the presence of parasites, sterile. However, this food source is not freely available, nor is obtaining it devoid of risk. It circulates inside vessels hidden underneath the skin of mobile hosts that are able to defend themselves and even predate the insects that try to feed on them. Thus, the haematophagous lifestyle is associated with major morphological, physiological and behavioural adaptations that have accumulated throughout the evolutionary history of the various lineages of blood-sucking arthropods. These adaptations have significant consequences for the evolution of parasites as well as for the epidemiology of vector-transmitted diseases. In this review article, we analyse various aspects of the behaviour of triatomine bugs to illustrate how each behavioural trait represents a particular adaptation to their close association with their hosts, which may easily turn into predators. Our aim is to offer to the reader an up-to-date integrative perspective on the behaviour of Chagas disease vectors and to propose new research avenues to encourage both young and experienced colleagues to explore this aspect of triatomine biology.

Key words: Triatominae - Triatoma - Rhodnius - behaviour - sensory ecology - cognition

Triatomine bugs (order Hemiptera, family Reduviidae, subfamily Triatominae) are a group of approximately 140 species, many of which are either actual or potential vectors of Chagas disease, the parasitic illness caused by the flagellate Trypanosoma cruzi. Most species feed on small terrestrial and arboreal mammals, especially didelphids, edentates and rodents. Others are associated with bats and some are associated with birds. Several species have colonised human dwellings, where they transmit the parasite to humans and domestic mammals. The most epidemiologically significant triatomines are the wideranging species Triatoma infestans, Triatoma dimidiata and Rhodnius prolixus (Lent \& Wygodzinsky 1979). Although Chagas disease was only discovered in 1909, its sylvatic cycle first developed millions of years ago as an enzootic disease of wild mammals. Its transmission to humans began accidentally in the form of an anthropozoonosis when men invaded sylvatic environments. Endemic Chagas disease became established as a zoonosis over the last 200-300 years due to deforestation for agricultural and livestock-rearing purposes and as a result of the adaptation of triatomines to exploit humans and their domestic animals as food sources (Coura \& BorgesPereira 2007). Even though some triatomine species are present in restricted regions of the Old World, Chagas disease is endemic to the Americas and represents a major sanitation issue in Central and South America.

\section{doi: 10.1590/0074-0276130409}

Financial support: ANR (EcoEpi project), CNRS, Le Studium, University of Tours, CNPq, FIOCRUZ, FAPEMIG

+ Corresponding author: claudio.lazzari@univ-tours.fr

Received 21 August 2013

Accepted 7 October 2013
Triatomines are hemimetabolous insects. Each of their five larval instars (and also including both male and female adults) is considered to be obligatorily haematophagous. The larvae require massive blood meals to trigger moulting. In turn, adults require significant amounts of blood for energy-consuming activities such as reproduction and dispersion. As a relic of their predatory origins, some species, if sufficiently hungry, are still able to feed on the haemolymph of other insects (Lent \& Wygodzinsky 1975, Lorosa et al. 2000, Garrouste 2009, Pontes et al. 2011).

The natural habitats of triatomines include palm tree crowns, hollow tree trunks, burrows, rock piles and other refuges, where they live in association with their hosts. They come into contact with humans in forested areas or when they arrive in more built-up areas by flying into and colonising human dwellings. Rural adobe houses typically present abundant crevices in which bugs can find shelter. Triatomines become infected with T. cruzi when they feed on infected mammals (Clayton 2010). The parasites then multiply in the insect gut and are later eliminated with the faeces, which serve as the vehicle for transmission to the next vertebrate host. When bugs feed on blood, they typically defecate on the skin of their host and, thus, deposit $T$. cruzi close to the wound, allowing it to penetrate into the skin (Clayton 2010). At the beginning of $T$. cruzi infection, the parasites can cause acute symptoms such as fever, swelling of the lymph nodes and skin lesions. This acute infection then evolves into a chronic one that can persist unnoticed for many years before causing cardiac or digestive disorders in roughly one-third of untreated patients, which will eventually lead to their death (Clayton 2010).

National campaigns aimed at the control of Chagas disease were initiated in the 1970s and significant multinational initiatives began in Latin America during the 1980s (Dias et al. 2002). Because there are no effective 
vaccines against $T$. cruzi, most efforts to control the disease focus on eliminating vector populations. These efforts involve the detection and extermination of vector species, which must be accomplished before they begin transmitting parasites in human dwellings.

In recent years, significant work has been devoted to the study of triatomine behaviour. This interest is not only inspired by the need to control these insects, but also because these bugs constitute an excellent model for the study of various fundamental aspects of haematophagy as well as for answering general questions concerning insect physiology, which have been considered since the time of VB Wigglesworth. This report presents an analytical review of current knowledge about triatomine behaviour and suggests possible future research topics addressing particular aspects for which a better understanding would be beneficial. In addition, we are convinced that triatomines, particularly $R$. prolixus, constitute excellent model systems for unravelling basic physiological and behavioural processes. The genome of $R$. prolixus is being revealed, which constitutes a major breakthrough in our comprehension of the genetic bases of the adaptations of insects to haematophagy as well as of the interaction of blood-sucking insects with parasites and hosts. The timing of this review is appropriate for opening novel research possibilities related to the functional genetics of insect behaviour on an updated basis.

Arrival in human habitats - The active dispersion of triatomines is influenced by seasonality, climatic conditions, the time of the day and an individual's physiological condition (Noireau \& Dujardin 2001, VazquezProkopec et al. 2004, Gurevitz et al. 2006, Barbu et al. 2010). Bugs travel from one sylvatic, peridomestic or domestic to another depending upon seasonal factors affecting host availability as well as population densities. In Yucatan, Mexico, T. dimidiata migrate from their sylvatic habitat into human dwellings and then back into the forest on a regular seasonal basis (Dumonteil et al. 2002, Barbu et al. 2009). Various studies have revealed that flying bugs captured in light traps typically present a poor nutritional condition (Noireau \& Dujardin 2001, Vazquez-Prokopec et al. 2006). However, recent captures performed using ground barriers have also revealed the dispersion of well-fed walking nymphs and egg-bearing females (Abrahan et al. 2011).

Similar to other nocturnal insects, these bugs are attracted to artificial lighting (Noireau \& Dujardin 2001, Vazquez-Prokopec et al. 2004, Carbajal de la Fuente et al. 2007), which lures them into human habitats (PachecoTucuch et al. 2012). This represents a paradox because triatomine bugs are reputed for their marked photophobia (Reisenman et al. 1998). Although the attraction of nocturnal insects to artificial lighting is extensively documented, the orientation mechanism underlying this attraction remains obscure in most cases. It is sometimes assumed that artificial lights, because of their relative proximity, out-compete the moon as a spatial reference when insects orientate by means of menotaxis (Jander 1963, Rich \& Longcore 2006), though this might not always be the case. The question of why photophobic triatomines are attracted to artificial lights has important epidemiological significance. The epidemiological consequence of erroneous menotaxis (i.e., using a lamp instead of the moon as a reference) is that only a fraction of dispersing bugs will eventually reach the light source. However, if the bugs were truly attracted by artificial lights, all of the dispersing individuals would actively move towards the bright spots contrasting against the dark background of the night, thus increasing the probability of their arrival in human dwellings. The analysis of flight initiation under controlled conditions in T. infestans and $R$. prolixus has revealed that the take-off bearing of bugs tends to point directly towards light sources (Minoli \& Lazzari 2006), supporting a true attraction. As menotaxis is not used to move towards or away from a light, but only to maintain a straight course, the bearing of each insect relative to the light source would be expected to vary from one individual to another and no preferred direction would be observed. The paradox of photophobic insects being attracted by light therefore appears to be solved. Nevertheless, a number of questions remain, such as those related to the switching of the sign of the phototactic tendency from negative to positive in dispersing bugs. Some issues that should be investigated to achieve full comprehension of this phenomenon include the influence of the particular stimulus pattern (i.e., sporadic light spots contrasting against the darkness of the night vs. fully illuminated environments), the type of locomotion (walking vs. flight) and/or the developmental phase (i.e., larvae vs. adults).

Choice of refuge - Upon arrival in a new habitat, triatomines must find adequate shelter to obtain protection against daylight and potential predators. Strategic refuge selection is also biologically important to find suitable microclimates in which to hide, moult and lay eggs and facilitates interactions with conspecifics to find symbionts and mates.

Natural (burrows, nests, hollow tree-trunks) and artificial (rural houses and peridomestic structures) habitats provide an abundance of shelters, although many of them do not offer suitable conditions. Shelters can be subject to high temperatures, humidity and intense illumination or may not be inhabited by colonies of conspecifics.

Guidance towards such refuges is assisted by volatiles emitted from triatomine excrement deposited through stereotypical behaviour that produces faecal-spot distribution patterns in the vicinity of access points to refuges (Lorenzo \& Lazzari 1996, Vitta et al. 2007). Excrement emits pheromones (see below) that attract bugs and, due to the proximity to refuges, constitute a preferred landmark in the overall chemical landscape (Lorenzo \& Lazzari 1996). To be useful, landmarks need to persist within the environment throughout consecutive activity cycles. However, to avoid attracting bugs towards uninhabited refuges, they must also have a transient effect. The average duration of the attractiveness of triatomine faeces is approximately 10 days (Figueiras \& Lazzari 2000). This time period appears to be sufficiently long to minimise maintenance efforts, but short enough to ensure that abandoned shelters cease to attract new individuals. 
As indicated above, triatomines exhibit intense negative phototaxis, showing a spontaneous tendency to avoid illuminated environments. Even though this behaviour may appear stereotyped, it is, in fact, finely modulated and demonstrates plasticity and adaptation. The factors affecting the expression of negative phototaxis are of both endogenous and exogenous origins and include circadian clocks (Reisenman et al. 1998), changes in eye sensitivity (Reisenman et al. 2002), interactions between inputs from compound eyes and ocelli (Lazzari et al. 1998, 2011), the spectral composition of the light (Reisenman \& Lazzari 2006) and, finally, bimodal integration between visual and olfactory inputs. The last factor is particularly important for the selection of refuges because light or darkness does not evoke the same response when aggregation pheromones are present vs. absent (Reisenman et al. 2000).

Once insects are inside a refuge, endogenous and exogenous mechanisms ensure akinesis (i.e., immobility) during the daytime because circadian clocks reduce the general activity of bugs (Lazzari 1992) (see below). Physical contact with the substrate and with congeners provides mechanosensory stimulation that triggers thigmotaxis (Wigglesworth \& Gillet 1934), i.e., reflex immobility triggered by the mechanical stimulation of a significant portion of the body surface. In addition, arresting factors derived from the cuticle also maintain immobility (see below).

Preferred shelters are those with amenable microclimates [in terms of temperature and relative humidity $(\mathrm{RH})]$ and triatomines express a clear thermo and hygropreference in their selection of such locations (Lorenzo \& Lazzari 1999). Triatomines appear to be more selective in terms of $\mathrm{RH}$ than temperature. In most species studied, a marked xeropreference has been verified. This response might be beneficial, as it may help to avoid infections by entomopathogenic fungi that tend to develop in humid environments (Roca \& Lazzari 1994, Guarneri et al. 2002). The thermopreference of triatomines is also marked and dynamic, varying from species to species. The preferred temperature varies continuously in accord with time elapsed since feeding and also as the day progresses (Lazzari 1991b, Pires et al. 2002a, Schilman \& Lazzari 2004, Guarneri et al. 2003). Immediately after feeding, the bugs remain at relatively high temperatures and move towards lower temperatures as the time of fasting increases. Thus, the bugs accelerate the conversion of acquired nutrients in their body mass and/or eggs. Subsequently, if contact with a new host is not possible, triatomines tend to slow their metabolism by moving to colder places (Lazzari 1991b). The daily thermopreference rhythm is discussed in more detail below.

In summary, refuge selection depends on the multimodal integration of external stimuli and endogenous signals.

Food foraging - During the first hours after the fall of dusk, triatomines end their akinesis and begin exploring their environment, searching for potential food sources, i.e., warm-blooded vertebrates. Food source detection is achieved via the detection of air currents carrying dis- tinctive odours, water vapour and heat. Host detection requires not only a high level of sensitivity, but also the capacity for integrating multimodal information. In addition, inhibitory mechanisms keep the insects away from hosts when a blood meal is not absolutely necessary.

Chemical cues - The detection of host odours stimulates triatomines and will induce them to leave their refuges. Appetitive searching and long-range orientation are then exhibited if appropriate environmental signals, such as host-associated cues, are perceived. When detected from a distance, olfactory cues do not provide directional information by themselves, but can trigger positive anemotaxis, i.e., the tendency to move in the opposite direction to air currents (Núñez 1982, Taneja \& Guerin 1995, Guerenstein \& Guerin 2001, Guerenstein \& Lazzari 2009). When chemical cues are detected in close proximity, they can stimulate insects and attract them. Similar to virtually every blood-sucking insect, carbon dioxide (CO2) modifies the behaviour of triatomines (Barrozo \& Lazzari 2004a, Guerenstein \& Hildebrand 2008). Triatomine responses towards $\mathrm{CO} 2$ are modulated by an endogenous circadian rhythm (Barrozo et al. 2004a, Bodin et al. 2008), leading the insects to respond only during the first hours of the scotophase. In fact, during this period, the motivation to feed is also higher (Lorenzo \& Lazzari 1998). The responsiveness of triatomines to $\mathrm{CO} 2$ is dependent upon their physiological condition as well. Long starvation periods do not strengthen the response (Barrozo \& Lazzari 2004a), however, recent feeding switches the effect of $\mathrm{CO} 2$ from acting as an attractant to a repellent (Bodin et al. 2009b). Moulting and reproduction (Bodin et al. 2009a, b) also affect the responses of bugs to $\mathrm{CO} 2$. In contrast to other haematophagous insects, especially several mosquito species (Bosch et al. 2000), CO2 is not an essential component involved in the attraction of triatomines, although it is sufficient to evoke such attraction (Núñez 1982, Guerenstein \& Guerin 2001, Barrozo \& Lazzari 2004a, b). Interestingly, other host odourants, such as nonanal, increase bug activity (Guerenstein \& Guerin 2001), while others that are found in human sweat (Cork \& Park 1996), such as isobutyric acid and 1-octen-3-ol, induce insect attraction (Guerenstein \& Guerin 2001, Barrozo \& Lazzari 2004b). Finally, ammonia, which is present in the sweat and urine of vertebrates, is able to evoke both the activation and attraction of T. infestans (Taneja \& Guerin 1997).

In nature, these insects are confronted by mixtures of odours, rather than isolated volatiles. The components of such mixtures often interact in a synergistic form. In the case of triatomines, the response threshold for pure $\mathrm{CO}_{2}$ is approximately $300 \mathrm{ppm}$ above the atmospheric concentration (Barrozo \& Lazzari 2004a). In contrast, L-lactic acid and short-chain fatty acids, which are present in human sweat and skin (Cork \& Park 1996, Bernier et al. 2000), are completely ineffective over a wide range of concentrations when presented alone (Barrozo \& Lazzari 2004a, b). Nevertheless, when sub-threshold amounts of $\mathrm{CO}_{2}$ are combined with L-lactic acid and fatty acids in the appropriate proportions, the attractiveness of the mixture becomes similar to that of a living 
host (Barrozo \& Lazzari 2004b). Despite the complexity of mixtures of odours emanating from vertebrates, few appear to be able to evoke strong attraction responses when presented in the correct proportions. Recently obtained experimental evidence also suggests that microorganisms associated with human skin may produce volatiles that are attractive to $R$. prolixus (Ortiz \& Molina 2010). This finding adds a novel dimension to the analysis of how Chagas disease vectors recognise and localise to their hosts.

Physical cues - Although both vision and vibroreception are well-developed triatomine senses, there is currently no evidence that they play a role in host orientation. The most important host-associated physical cue perceived by these bugs is heat. While the heat emitted by host bodies is used as an orienting cue for many bloodsucking insects, its role remains relatively unknown for the vast majority of these insects (Lazzari 2009). In contrast, the thermal sense of triatomines has been the subject of detailed analyses. The sensitivity of triatomines to heat is extremely high, such that the insects can detect differences in thermal energy on the order of a few $\mu \mathrm{Watts} / \mathrm{cm}^{2}$ (Lazzari \& Núñez 1989b, Lazzari 2009). It has been calculated that $T$. infestans is capable of detecting the heat emitted by a human face from a distance of approximately $2 \mathrm{~m}$ and by a mammal the size of a dog from several metres away (Lorenzo et al. 1999a).

Triatomines remain the only group of blood-sucking insects whose ability to perceive host-emitted infrared radiation has been demonstrated (Lazzari \& Núñez 1989b, Schmitz et al. 2000). This ability has important implications for successful host finding because the propagation of infrared radiation is not disrupted by air currents (which do disrupt conductive and convective heat transfer) or by the relative position of the insect with respect to the thermal source (which does influence convective heat transfer) (Guerenstein \& Lazzari 2009, Lazzari 2009).

A remarkable characteristic of $T$. infestans is its ability to detect the temperature and distance of heat sources, independent of their size, based on thermal information alone. This behaviour appears to be possible due to the capacity of these insects to perceive radiant heat (Lazzari \& Núñez 1989b, Lazzari 2009). A hypothetical model of how these bugs distinguish the temperature, size and distance of objects based on thermoreceptive inputs and active antennal movements has been proposed by Lazzari (2009). This model provides an explanation for the ability of triatomines to discriminate distant (or small) burning objects from closer (or large) tepid ones, even if the amount of thermal energy reaching the insect antennae is of the same magnitude.

The extreme thermal sensitivity of triatomines, together with their ability to discriminate the temperature of distant objects (Lazzari 2009), poses the question of whether the absolute temperature of a heat source or the difference between the temperatures of objects and their background are, in fact, used by these insects to recognise their hosts. This question is related to another, more practical one: are triatomines able to perceive objects with the temperature of a host when the ambient tem- perature is higher? Experiments have been performed to test the response of bugs to an object presented at different temperatures in a chamber providing a background where the temperature could also be modified (Fresquet \& Lazzari 2011). The bugs responded by trying to bite objects with temperatures between $30-40^{\circ} \mathrm{C}$, but only if the surrounding environment was colder than the objects themselves. Therefore, their ability to measure the temperature of objects is only effective when a positive difference exists between the object and the air temperature (Fresquet \& Lazzari 2011).

Notably, in a natural context, triatomines are not exposed to single cues, such as specific odours or heat, but to multiple cue combinations of cues including different sensory modalities (chemical, thermal, hydric). The extreme heat sensitivity of triatomines may be further enhanced by its integration with cues from other sensory modalities. For example, it has been demonstrated that water vapour, which constitutes a close-range orientation cue by itself, also increases the responsiveness of triatomines to heat (Barrozo et al. 2003). However, it is still unclear whether this is due to the convergence of different sensory inputs in the insect brain or to a physical phenomenon (i.e., moist air transports more heat that dry air). The Figure summarises all of the endogenous and exogenous factors affecting host-seeking and host-feeding among Chagas disease vectors. These data strongly suggest that bug responses to the presence of a potential host depend on the multimodal integration of a variety of external cues, the insect's physiological state and its individual experience (see below).

Locating blood vessels - In addition to its role as an orienting signal, heat is used to locate blood vessels hidden underneath the skin (Ferreira et al. 2007). By analysing the feeding behaviours of bugs on a live host, it has been demonstrated that insects do not bite randomly. Instead, they extend their proboscises directly towards vessels. When host skin was replaced with a vessel-shaped heat source on a heated metal plate, where the temperature of the two heat sources were controlled independently, the same precision in detecting the warmest area was observed, suggesting that heat discontinuities over the skin surface area are used to guide biting behaviour. Biting the warmest patch of the skin surface requires bilateral integration of the thermal inputs provided by the two antennae. If this integration is experimentally altered via unilateral or bilateral antennectomy, the bugs miss the target or are unable to exhibit any proboscis extension response (PER), suggesting that, if present, rostral thermoreceptors are not involved in guiding biting behaviour (Ferreira et al. 2007).

Finally, it is worth mentioning that heat is a very important factor in finding and biting hosts; however, once a blood vessel has been contacted, heat is no longer important. Food recognition in triatomines is based exclusively on two properties of the food: its osmolarity (Guerenstein \& Núñez 1994) and the presence of phagostimulant compounds (Friend \& Smith 1977). If the antennae are stimulated by heat stimulation to evoke a PER, bugs can be induced to feed on blood as cold as 


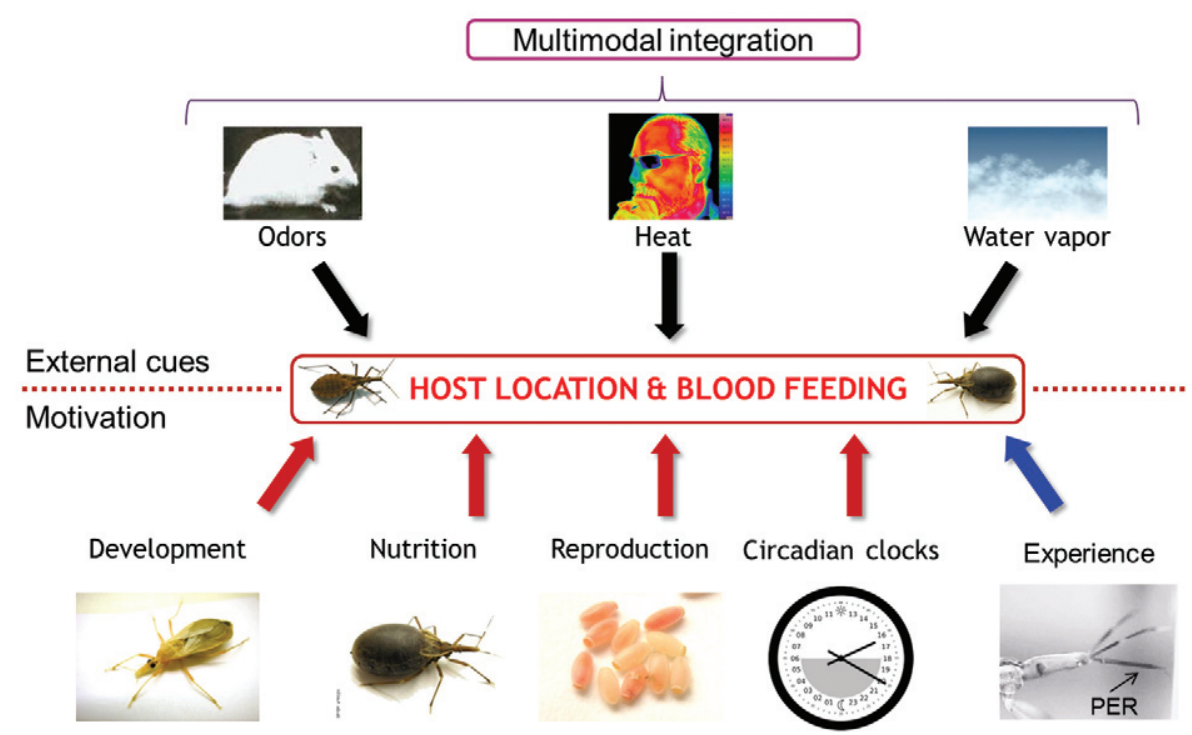

Exogenous and endogenous factors influencing host-seeking and feeding behaviour in Chagas disease vectors. Hosts multimodal cues which attract or repel insects depending on their motivational state. PER: proboscis extension response.

$3^{\circ} \mathrm{C}$, although the time required to obtain a full meal may be 2-3 h (Lazzari \& Núñez 1989a). This observation demonstrates that each step of the feeding behaviour depends on particular sensory cues, which act in a precise sequence.

After locating and identifying a blood source, the insect must begin ingestion, transferring the blood into its midgut. For this purpose, insects use suction pumps located along their head. Essentially, these pumps create negative pressure between the opening of the food canal and the lumen of the alimentary compartment through the contraction of powerful muscles occupying most of the volume of the head in triatomines (Bennet-Clark 1963, Lehane 2005). The development of an ingestion pump was necessary to compensate for the need to maintain a relatively narrow alimentary channel, to minimise the tissue damage produced in the host during blood feeding, reducing its probability of being detected and killed.

Insect-related parameters include the negative pressure produced by the ingestion pump, the dimensions of the food canal, host blood viscosity and the size of red blood cells and their capacity to deform and influence the ingestion rate. With regard to the hosts, the rheological and haematological characteristics of the blood vary across vertebrates, particularly the size of the erythrocytes and the erythrocyte aggregation behaviour and plasma and whole blood viscosities are likely crucial factors influencing the performance of a given insect when it feeds on different host species (Kingsolver \& Daniel 1995, Pereira et al. 2006).

Communication - Triatomines use both vibratory and chemical mechanisms of communication. Stridulation is employed for sexual and defence-related communication, whereas pheromones are used to communicate messages in several behavioural contexts.
Vibration signals - The ability of triatomines to stridulate was recognised long ago (Readio 1927, Schofield 1977). Some species of this subfamily are able to produce sounds that are audible to man; however, the biologically relevant cue is actually vibratory (i.e., waves propagating through the insect body and through the substrate), rather than the sound produced, which is an epiphenomenon. This is because triatomines do not have auditory organs and that no behavioural evidence indicates that they respond to airborne sounds (Manrique \& Lazzari 1994, Roces \& Manrique 1996, Manrique \& Schilman 2000, Lazzari et al. 2006).

Triatomines produce vibrations by means of a stridulatory organ located on the ventral prothorax of larvae and adults (Lent \& Wygodzinsky 1979). Stridulation occurs when the insect rubs the tip of its rostrum against a prosternal groove, scraping across cuticular ridges with alternate back and forth movements (Schofield 1977).

Initial reports of stridulation in triatomines described this phenomenon in bugs being grasped by forceps (Schofield 1977). In this particular situation, adults of both sexes as well as larvae from all instars are able to stridulate. However, the spontaneous occurrence of stridulation in untethered bugs has only been observed during mating activities (Manrique \& Lazzari 1994, Lazzari et al. 2006). Non-receptive females reject male copulatory attempts by displaying various evasive behaviours, including stridulation (Manrique \& Lazzari 1994). Although differences have been reported in the mating behaviours of various triatomine species, stridulation by unreceptive females during male mating attempts appears to be a frequent feature (Lazzari et al. 2006).

The male-deterring stridulation of female $T$. infestans consists of a long series of repetitive syllables, each of which is composed of two chirps, whereas that of $R$. prolixus is characterised by syllables consisting of a 
single chirp. As indicated above, larvae, males and females of both species also exhibit "disturbance" stridulation when they are clasped with forceps. The vibratory signals emitted in this context differ in their frequency spectra from those emitted during mating, even when they are produced by the same individual (Roces \& Manrique 1996, Manrique \& Schilman 2000). Therefore, triatomines are able to emit different vibratory signals as a function of the biological context.

Comparative analysis of the disturbance signals emitted by $T$. infestans, Triatoma guasayana, Triatoma sordida, $R$. prolixus and Dipetalogaster maxima offers relevant information on their potential function (Schilman et al. 2001). The vibrational signals produced by all of these species show similar frequency spectra and repetition rates, even though these insects differ in size and their stridulatory grooves display different inter-ridge distances. Analysis of these frequency spectra shows that the main carrier frequency of the vibratory signal presents a value of approximately $2,000 \mathrm{~Hz}$. The temporal structures of the distress signals of all five species display repetition rates of approximately eight syllables. To be effective, the vibratory signals produced in different behavioural contexts should match the reception capacities of a receiver and show a specificity corresponding to their biological significance. In this regard, only female male-rejecting stridulation appears to be within the sensory range of triatomines, with the frequency of perturbation stridulation being too high to be perceived by the bugs (Lazzari et al. 2006). Disturbance stridulations likely constitute a defensive strategy employed against predators, rather than a signal for intraspecific communication (Schilman et al. 2001, Lazzari et al. 2006) because (i) males, females and larvae of all of the analysed species produce stridulations with similar characteristics, (ii) disturbance stridulations present greater similarity among different species than the two types of stridulation produced by an individual female, (iii) no sensory organs are known to exist in triatomines that are capable of detecting high-frequency vibratory signals, (iv) stridulation is a common strategy employed by many insects in an attempt to induce predators to release them and (v) in adults, disturbance stridulation is accompanied by the emission of pungent secretions from their Brindley's glands.

Chemical signals - The chemical communication of triatomines has been a major topic of inquiry in the past several years. The use of pheromones as agents for behavioural manipulation is a successful method employed against other pests and could also be an alternative for the control of Chagas disease vectors (CruzLopez et al. 2001). Different types of pheromones have been identified in triatomines that mediate aggregation, arrestment, mating and alarm warning (Baldwin et al. 1971, Schofield \& Patterson 1977, Ward 1981, Figueiras \& Lazzari 1998b).

Aggregation and arresting pheromones - The faeces of triatomines are a source of aggregation pheromones that attract and aggregate bugs in their proximity (Schofield \& Patterson 1977, Lorenzo Figueiras 1994). It was originally suggested that the function of these signals might be to indicate a suitable food source (Schofield \& Patterson 1977). Further studies revealed the accumulation of faeces on the access paths to refuges and the role of their presence in the selection of artificial shelters suggests a major role as chemical landmarks for finding shelters (Lorenzo \& Lazzari 1996). The pheromones present in excrement are not species specific in terms of their effects and are capable of attracting bugs of other species (Figueiras \& Lazzari 1998a, Pires et al. 2002b). They can be extracted using polar solvents and chemical analyses have revealed multiple compounds whose biological roles are not yet clear (Cruz-Lopez et al. 2001, Alzogaray et al. 2005).

Another signal promoting aggregation is deposited by the bugs on substrates and remains as a "footprint". This signal has a different chemical composition from that of faeces and can be extracted with non-polar solvents. Receiver insects require physical contact for detection (Figueiras \& Lazzari 1998b) and, coincidently, these signals appear to be composed of a mixture of hydrocarbons of cuticular origin (Lorenzo Figueiras et al. 2009). Therefore, these pheromones can be considered to act as an arresting factor, rather than an attractive one.

Sexual pheromones - The study of the sexual pheromones of triatomines has been particularly difficult with respect to their origins, biological significance and composition. Despite a considerable amount of experimental work and published observations (Cruz-Lopez et al. 2001), only recently has some light been shed on the subject. The first piece of evidence of the likely occurrence of these pheromones in triatomines was obtained in R. prolixus (Baldwin et al. 1971) and, much later, in $T$. infestans (Manrique \& Lazzari 1995). In these species, mating couples release volatiles that attract and aggregate males that eventually mate with the same female.

The origin of sexual volatiles has been a matter of controversy due to the presence of isobutyric acid produced by Brindley's glands and found in the "headspace" of mating pairs (i.e., the air surrounding them) (Fontan et al. 2002). This compound is released when bugs are perturbed (Barrett et al. 1979) and experiments occluding the glands of males and females have revealed that the metasternal glands (MGs) of females are the source of the sexual pheromones (Crespo \& Manrique 2007). Chemical analysis has confirmed that isobutyric acid is not present in MGs (Manrique et al. 2006). Recent evidence suggests that female MG odours are preferentially emitted during the scotophase (Pontes et al. 2008), are capable of inducing males to leave their shelters (Pontes 2010) and take flight (Zacharias et al. 2010) and are orientated toward airstreams associated with female odours, displaying positive anemotaxis (Vitta et al. 2009, MayConcha et al. 2013). The contents of these glands present complex mixtures of ketones, alcohols, dioxolanes and aldehydes (Manrique et al. 2006, Pontes et al. 2008, Vitta et al. 2009, May-Concha et al. 2013).

Apparently, cuticular hydrocarbons may also play a role in mating (Cocchiararo-Bastias et al. 2011). An integrative and critical analysis of old and new evidence 
is still necessary to develop a complete picture of how chemical communication mediates the sexual behaviour of triatomines. In addition, laboratory and field experiments should be performed to unravel the adaptive role of the apparent polyandry observed in several species of these bugs.

Alarm pheromones - As indicated above, triatomines release a pungent odour when disturbed. These secretions are composed of isobutyric acid and a complex mixture of other volatiles (Cruz-Lopez et al. 1995, Guerenstein \& Guerin 2004, Manrique et al. 2006) produced by the Brindley's glands. Triatomines respond to high concentrations of isobutyric acid by walking away from the source (Manrique et al. 2006), whereas they approach sources releasing low quantities (Ward 1981, Guerenstein \& Guerin 2001). This dual response has not facilitated distinction of the biological significance of this compound, as discussed in the previous section. Its release in the presence of danger, in addition to its repulsive role, has allowed us to recognise the Brindley's gland secretion as an alarm pheromone. What is not fully clear, however, is its functional role. Both types of glands, Brindley's and metasternal, are only found in adults. Since the latter are involved in sexual communication, its imaginal nature seems to be a logical conclusion. Nevertheless, the fact that nymphs can respond to, but not produce alarm pheromones, is less easy to understand in adaptive terms. The plasticity of the response of bugs to alarm pheromones (Minoli et al. 2013) adds another intriguing component that deserves further analysis.

Parsimonious use of chemical information - An integrative examination of the chemical ecology of triatomines reveals that they possess a complex pheromonal system that is quite different from that of classical textbook examples. The triatomines represent a group of insects that are at a sufficient phylogenetically distance from the classical model species, e.g., moths, beetles and social hymenoptera, that independent evolution of their pheromonal communication system can be hypothesised.

Another interesting conclusion derived from this idea is that the same compounds appear to be related to chemical signalling with very different biological meanings. For example, isobutyric acid has been found in Brindley's gland secretions, bug excrement and host-emitted volatiles (2-butanone in the Brindley's and MGs). Acetic acid is present in alarm pheromones, bug excrement and host odour blends. Thus, in contrast to other insects, it appears that specific biological triggers, such as sexual partnering, searching for refuge and the presence of danger and/or food, are not associated with specific compounds in triatomines but, rather, with particular combinations of chemical signals, thus defining the value of each mixture. This would involve the parsimonious use of sensory information in the sense that the same set of sensory organs is involved in the detection of different resources. They do not appear to function in the traditional manner of generalist receptors, i.e., in recognising portions of molecules, but by informing the brain of the presence of specific components whose simultaneous occurrence defines a particular biological context. This type of system would be favoured by natural selection due to its economy in terms of the diversity of sensory receptors and centralised information processing.

The state-dependency of the response to host cues can also be considered to be an example of a parsimonious use of sensory information. As indicated above, the same cues that attract bugs motivated to feed also repel engorged bugs or those that are ready to moult (Bodin et al. 2009a, b). In this case, the integration of external and internal cues determines their interpretation, revealing the proximity of warm-blooded animals, which will then be considered either a potential food source or a predator and may evoke an adaptive response, i.e., attraction or avoidance. Again, the same information may have different biological meanings according to the physiological state of the insect (Bodin et al. 2009b, Reisenman et al. 2013).

Temporal organisation - A salient characteristic of the biology of triatomines is their marked temporal patterns. It is known that selective pressures have acted to adjust the biting activity of blood-sucking insects to the time of the day when hosts are less active (Barrozo et al. 2004b). However, the degree of synchronisation of the various behavioural and physiological processes that have been proven to occur in triatomines is quite unusual. This temporal arrangement begins at a very early stage in the life of these bugs (Lazzari 1991a). Even "once-in-a-lifetime" events in triatomine biology are controlled by circadian clocks. First-instar larvae hatch from eggs in the early morning, when the environmental RH reaches a daily maximum. Nevertheless, this event is not triggered by environmental changes, but by an endogenous circadian clock (Lazzari 1991a, Schilman et al. 2009). Ecdysis occurs during the same temporal window across the various instars. Again, a circadian clock has been proven to control this process (Ampleford \& Steel 1982). The temporal synchronisation of egg hatching and ecdysis has an evident adaptive value that is likely related to the deleterious effect of low RH on these processes. As indicated above, triatomines exhibit a marked xeropreference and this preference is not modified during egg-laying activity or moulting (Roca \& Lazzari 1994, Guarneri et al. 2002). Thus, we can conclude that triatomines exhibit a temporal hygropreference, rather than a spatial one to adapt their needs at critical moments of their life. Instead of moving to humid places to perform humidity-sensitive activities, they perform them in specific phases of the day that typically present high humidity.

Even though triatomines are frequently described as nocturnal insects, their general spontaneous activities can be categorised as bimodal, provided that they are split into two different temporal windows: one that encompasses the first hours after dusk and a second during the first hour after dawn, each of which is controlled by a different internal oscillator (Lazzari 1992). These two peaks of activity comprise all of the activities displayed by these bugs, including the modulation of related sensory sensitivities. As a result, part of these processes take place during the first activity period, e.g., host search- 
ing, feeding, departure from refuges, egg laying and dispersion by flight, whereas others occur during the second activity peak, such as ecdysis, egg hatching, refuge searching and bug aggregation (Constantinou 1984, Barrozo et al. 2004b).

The behavioural response to external stimuli is also rhythmically modulated, as demonstrated by the fact that the sensitivity of the insects is higher when a given signal becomes biologically relevant. For example, the eyes become more sensitive to light during the night, thus enhancing the negative phototactic response of bugs (Reisenman et al. 1998, 2002). The responsiveness to odours also varies in a highly specific manner, with the response to host odours being maximal during the nocturnal activity peak, whereas the response to aggregation pheromones is maximal during the early morning peak. These modulatory changes match the time of the day in which each olfactory signal is biologically relevant, maximising its efficiency (Barrozo et al. 2004a, Bodin et al. 2008).

A rhythmic change in the thermopreference of bugs has been described in several triatomine species, leading the bugs to expose themselves to relatively higher temperatures at the beginning of the night, before displaying their first activity peak. In the early photophase at the end of the second peak, the bugs return to cooler places. This rhythm is also controlled by an internal circadian clock (Lazzari 1991b, Minoli \& Lazzari 2003). It has been suggested that this thermopreference rhythm is intended to increase the body temperature of the bugs and, in turn, their metabolic rate just prior to the initiation of their regular daily activity. Conversely, both body temperature and metabolism are reduced during resting periods.

In summary, many triatomine activities and processes are modulated in the form of daily rhythms. Most are expressed at the individual level (e.g., activity, oviposition) whereas others take the form of population rhythms (egg hatching and ecdysis). Interestingly, all daily rhythms have an endogenous basis and are controlled by circadian clocks, with the exception of the modulation of aggregation pheromone sensitivity (Bodin et al. 2008) and the adaptation of ocelli to light (Lazzari et al. 2011). The precise temporal allocation of every type of sensitivity and action would be sufficiently significant to lead to the development of dedicated, specific clocks via natural selection. The fact that each activity shows a particular temporal window is the result not only of its association with one of the two activity periods, but also of the temporal adjustment of that activity to a particularly appropriate temporal context, e.g., the host's resting period. Research on the chronobiology of triatomines also contributes to our understanding of the structure of the insect clock and the manner in which it coordinates different physiological processes (Steel \& Vafopoulou 2006, Vafopoulou et al. 2007, 2010), again revealing the value of these bugs as model systems for analysing fundamental biological questions.

One important consequence for researchers in relation to this strong temporal organisation of triatomine physiology and behaviour is the conclusion that any process of interest must be studied in its appropriate tempo- ral context. If the proper timing is not observed in these types of studies, the results obtained may be biased, thus masking significant patterns (Lazzari et al. 2004).

Learning and memory - For quite some time, insects were considered to be "reflex machines", whose behaviours were mostly ruled by stereotyped, innate responses to external stimuli (Giurfa 2004). The cognitive abilities of insects are now widely recognised and their tiny brains have been proven to be capable of complex forms of learning, including the acquisition of rules and concepts (Giurfa et al. 2001, Giurfa 2003). Although learning and memory are major issues in the study of animal behaviour, most studies in this arena have focused on a restricted subset of insect species (particularly on Drosophila and honeybees), none of which displays a haematophagous lifestyle.

The potential cognitive abilities of blood-sucking insects may have significant epidemiological consequences because they can be responsible for the development of heterogeneous biting patterns within host populations (Kelly \& Thompson 2000, Kelly 2001). Due to the strong selection pressures to which blood-sucking insects are exposed and based on the fact that they must obtain their food from mobile and defensive hosts, it is reasonable to consider whether they present well-developed cognitive abilities. The capacity to learn to recognise the most vulnerable hosts could allow individual experience to develop in blood-sucking insects, which could influence host selection and, hence, affect parasite transmission (McCall \& Kelly 2002).

Many field and semi-field studies have demonstrated behavioural responses that might indicate learning capabilities in disease vectors. Nevertheless, only a few controlled studies have succeeded in demonstrating conclusive experimental evidence of such capabilities (Alonso et al. 2003, Alonso \& Schuck-Paim 2006). In Chagas disease vectors, early experiments failed to demonstrate learning capabilities (Abramson et al. 2005, Aldana et al. 2005, 2008). In most cases, the difficulty of obtaining positive results can be associated with a series of issues that often plague these types of studies, including the lack of rigorous control over experimental variables (insect motivational state, for example), a misunderstanding of the concept of a "neutral stimulus" (using conditioning stimuli such as odours that cannot be perceived by the insect) and, finally, insufficient knowledge of the experimental model. It should also be noted that experimental psychology imposes a strict theoretical framework and requires numerous control experiments to properly assess the learning capabilities of an animal. In contrast to the study of other insects, such as honeybees and Drosophila, this framework is not properly followed in most studies involving disease vectors (Alonso \& Schuck-Paim 2006).

One major difficulty in the conditioning of bloodsucking insects, especially those whose diets are strictly composed of blood, is providing controlled quantities of food as a positive reward. In contrast to nectar feeders, which can be offered drops of sugar solution, most haematophagous insects must pierce the skin (or a mem- 
brane) to obtain their reward. In addition, these insects must be allowed to obtain precise amounts of blood to control their motivational state and rewards. Furthermore, it is necessary to design experiments in which an unconditioned stimulus is presented to induce insects to bite. Finally, a neutral stimulus, which has to be perceived by the insects, should be temporarily paired with this presentation (Vinauger et al. 2011a).

Triatomines appear to be good models for studying the cognitive capabilities of blood-sucking insects because (i) they can be reared in the laboratory under controlled conditions using artificial feeders, (ii) they are obligatorily haematophagous throughout their entire lifecycles, thus facilitating experimentation with juveniles and excluding potential interference of reproductive behaviour with the bugs' motivation to respond to the proposed stimuli, (iii) their size facilitates work under constrained conditions (i.e., fixation to a support or to a locomotion compensator), making precise control of stimulation protocols possible and (iv) a response that is widely exploited in honeybee and Drosophila learning studies, e.g., the "proboscis extension reflex", or PER, is expressed by these insects under thermal stimulation.

In recent years, the adaptation of olfactory conditioning protocols for appetitive and aversive training with $R$. prolixus has made it possible to demonstrate dual-conditioning responses to the same neutral odour (Vinauger et al. 2011a, b), i.e., the bugs were able to associate lactic acid (which does not evoke any oriented behavioural response when presented alone or after preexposition in naïve bugs) with the possibility of obtaining food or being exposed to punishment. When confronted with the choice between two air currents, one loaded with lactic acid, while the other was clean, the bugs either displayed significant attraction or significant repulsion to the lactic acid-laden current, depending on their individual experience. This outcome demonstrates the ability of bugs to learn and to remember information, but also their ability to use that knowledge in different contexts, e.g. use in orientation tests in an olfactometer, what they have learnt when confronted to an artificial feeder (Vinauger et al. 2011a, b).

The ability of triatomines to modify their responses to chemical stimuli as a function of their individual experience has been proven to affect their host choice (Vinauger et al. 2012). Hence, by inducing the choice of less-defensive hosts, the cognitive abilities of disease vectors may affect the distribution of parasites among them.

Recently, another classical learning paradigm in insects has been validated in triatomines, i.e., conditioning of the PER (Vinauger et al. 2013). This simple and well-characterised response, widely exploited in studies on bees, flies, butterflies and bumblebees, constitutes a powerful tool for unravelling neural mechanisms and characterising insect learning and memory. In the case of triatomines, PER-aversive conditioning has revealed basic aspects of the learning abilities of $R$. prolixus, indicating that it is able to remember information learnt $72 \mathrm{~h}$ earlier (Vinauger et al. 2013).

Another recent investigation indicated that pre-exposure modifies the subsequent behavioural response of $T$. infestans to the alarm pheromone and its main component (Minoli et al. 2013), with the effect being modulated by the previous time of exposure of the insects to the stimulus.

Behavioural manipulation - Controlling disease vectors by exploiting their behaviour requires solid knowledge of their biology. Information such as the identity of relevant attractive cues, the profiles of preferred shelter environments and estimates of species' ability to escape from traps is potentially useful in planning sampling, detection and killing activities designed for the control of these insects. It is also critical that the signals used to attract the insects as well as the context in which they are presented (i.e., the environment and timing), represent a combination that is biologically coherent to the insect. It is not simply a matter of combining all potentially attractive stimuli (pheromones, host odours and light) because any combination must be significant for the insect. Here, the "biological context" indicates a clear message (i.e., host, refuge, partner), an appropriate spatial location (e.g., indoors, outdoors, ground, walls, roofs) and temporal matching of the proposed information to the insect's needs (e.g., the timing of host searches or refuge localisation).

Two different aspects of the biology of Chagas disease vectors have been exploited to develop two methods of behavioural manipulation: capture traps and artificial refuges. In addition to light-traps, specific methods for trapping blood-sucking insects are predominantly based on the delivery of host-associated cues. The nature and source of attraction are quite different depending on the sampling conditions and the targeted species. The most efficient method, as would be expected, is the use of live hosts, typically consisting of a mouse or baby chicken (Noireau et al. 1999, 2002, Abad-Franch et al. 2000). The use of live hosts as bait can sometimes be impractical due to the difficulty of providing them with adequate care during extended field campaigns. Some alternative types of bait have been proposed and have already passed both laboratory and field testing. The simplest is cultures of Baker's yeast (a simple mixture of yeast, sugar and water) that produce $\mathrm{CO} 2$ and other volatiles, which are attractive for haematophagous insects (Guerenstein et al. 1995, Lorenzo et al. 1998, 1999b, Pires et al. 2000, Pedrini et al. 2009, Smallegange et al. 2010). More recently, a relatively simple combination of multimodal cues, including heat, water vapour, $\mathrm{CO} 2$ and short-chain fatty acids, has been used (Ryelandt et al. 2011). Both types of bait described above deliver compounds recognised to be attractive to many haematophagous arthropods and are useful for capturing not only blood-sucking bugs, but also mosquitoes and ticks (Smallegange et al. 2010, Ryelandt et al. 2011).

A major constraint of some devices is the short persistence of the attractive lure. Live hosts cannot be maintained for long periods of time inside small traps without food and water, while yeast cultures are cheap and very simple, but remain active for only a few hours and the chemical production of heat and/or $\mathrm{CO} 2$ by these cultures can only be promoted for a limited time. The peri- 
od of activity of yeast cultures can be prolonged through the use of an electric heater and/or gas bottles; however, these activities restrict the utility of such cultures to sites with electricity and increase the weight and costs of field equipment. For these reasons, traps exploiting hostsearching behaviour are short-term devices that are useful for detecting the presence of the bugs in the wild and indoors as part of sampling procedures. Recently, some devices have combined baits with killing or trapping devices (Pedrini et al. 2009, Rojas de Arias et al. 2012).

Artificial refuges operate in a different manner than baited traps. Rather than attracting bugs, they provide convenient resting places for them. Due to their physical structures, which induce bugs to enter these refuges and remain inside, they can even be used without chemical bait. Eventually, the bugs will produce "their own bait" when their excrement, containing aggregation pheromones, starts to accumulate, attracting other bugs searching for shelters. Cardboard boxes simulating refuges have been used as a part of the intra-domiciliary surveillance of Chagas disease vectors in endemic areas of Latin America (Gomez Núñez 1965, Wisnivesky-Colli et al. 1987). Other refuge-like devices composed of simple, resistant materials have also proven to be effective for outdoor bug detection (Vazquez-Prokopec et al. 2002). Currently, none of these devices includes artificial lures, however, this possibility deserves further exploration.

Synthetic triatomine pheromones could be used to interfere with intraspecific bug communication in sexual, alarm and aggregation contexts. Although these alternatives could constitute good options and despite the steadily advancing knowledge in this field, the use of synthetic pheromones for triatomine behavioural manipulation will require further research efforts.

Future issues and research needs - Relevant aspects of triatomine biology requiring further research can be identified based on our current knowledge. Moreover, further investigation could provide important insight into fundamental aspects of haematophagy and aid in the development of novel control tools. In addition, the $R$. prolixus genome sequence that has recently been made available has provided an extraordinary opportunity to look deeper into the genetic and molecular bases of haematophagous behaviour, further verifying this species as one of the most useful and fascinating models available in insect science.

Dispersion - Two important questions remain to be answered. The first is the role of signals (other than light) as navigation aids in finding host habitats. These signals would include odours dispersed by wind in particular. The second concerns the light orientation mechanism of the bugs, especially in regard to whether they display true attraction to light or erroneous menotaxis. As discussed above, this information is highly relevant from an epidemiological point of view.

Parasite behavioural impacts - It is well known that many parasites modify the physiology and behaviour of vertebrate hosts and insect vectors to their own advantage (Lehane 2005, Schaub 2006). The behaviour of parasitised bugs is a major issue for which only very restricted data are available. T. cruzi infections appear to have physiological consequences for the insects (Vallejo et al. 2008) and it is highly probable that their behaviour is also affected. For example, it has been demonstrated that the feeding behaviour of the bugs is modified by these infections (Botto-Mahan et al. 2006), but the impact of infection on most other behavioural traits remains unclear (Takano-Lee \& Edman 2002).

Sensory physiology - Some efforts have been made to better understand the sensory mechanisms involved in the detection and treatment of environmental information. Thermosensation, olfaction, mechanoreception, hygroreception and vision are sensory capacities involved in crucial aspects of a bug's life. The availability of novel electrophysiology, optophysiology and functional genetics methods provides a significant number of novel research possibilities for better understanding how haematophagous insects acquire and make use of information for locating resources and communicate.

Multimodality - A significant amount of information has been published on the response of many blood-sucking insects to host cues. Nevertheless, a very limited amount of data concerning multimodal sensory integration is available. It should be remembered that hosts emit diverse physical and chemical cues, many of which can be detected by the sensory systems of these insects. To understand how these vectors analyse their environment and use the available information to locate resources, it is necessary to understand how their integrative processes handle the various sensory modalities exploited and how multimodal sensory inputs interact to modulate the behaviour of these insects. This information would be critical in the development of innovative tools that manipulate triatomine behaviour.

State dependency - As indicated above, research in triatomines has provided important insight into how feeding behaviour is modulated by reproduction, metamorphosis, nutrition and circadian rhythms. The manner in which these factors interact with internal and external cues is beginning to be understood. However, significant work is still required to fully understand the motivational factors influencing different behaviours. Pending concepts include uncovering how these modulatory processes are effected, which molecules are responsible for signalling of the different known motivational states and whether these changes are implemented in the peripheral or/and central components of the nervous system.

Cognition - Determining the impact of the cognitive abilities of haematophagous insects on the transmission of parasites will require much more experimental and theoretical work. The cognitive sciences provide a solid and well-defined framework and classical insect models, such as honeybees and Drosophila, have served to validate experimental protocols for the analysis of learning and memory. Investigation of the cognition of disease vectors requires rigorous experimentation and appropriate controls to avoid incurring past mistakes. 
Chemical ecology - Despite the efforts made thus far, many aspects related to the use of odours still require clarification. It is necessary to determine the composition of the pheromones mediating the various behaviours mentioned above and the biological functionality of communication signals still needs to be better elucidated. The fact that pheromonal components of different natures and origins can be associated with the same biological context, such as assembly and sexual interactions, raises further questions as to how these factors operate in combination. Thus, much more work still needs to be done regarding chemical identification as well as behavioural analysis and experimentation.

\section{ACKNOWLEDGEMENTS}

To the work of many colleagues, who could not be included in this review because of space restrictions, to the Brazilian programs Ciência sem Fronteiras and INCT de Entomologia Molecular, as they have made this joint reflexion possible, and to the editors, for inviting us to participate of this special issue devoted to the memory of our colleague Alexandre Afranio Peixoto.

\section{REFERENCES}

Abad-Franch F, Noireau F, Paucar A, Aguilar HM, Carpio C, Racines J 2000. The use of live-bait traps for the study of sylvatic Rhodnius populations (Hemiptera: Reduviidae) in palm trees. Trans $R$ Soc Trop Med Hyg 94: 629-630.

Abrahan LB, Gorla DE, Catala SS 2011. Dispersal of Triatoma infestans and other Triatominae species in the arid Chaco of Argentina - Flying, walking or passive carriage? The importance of walking females. Mem Inst Oswaldo Cruz 106: 232-239.

Abramson CI, Romero ES, Frasca J, Fehr R, Lizano E, Aldana E 2005. Psychology of learning: a new approach to study behavior of Rhodnius prolixus Stål under laboratory conditions. Psychol Rep 97: 721-731.

Aldana E, Abramson CI, Lizano E, Vegas R, Sulbaran-Romero E 2008. Learning and orientation to odor in the bug Rhodnius prolixus Stål 1859 under laboratory conditions. Parasitol Res 103: 587-594.

Aldana E, Otalora F, Abramson CI 2005. A new apparatus to study behavior of triatomines under laboratory conditions. Psychol Rep 96: 825-832.

Alonso WJ, Schuck-Paim C 2006. The 'ghosts' that pester studies on learning in mosquitoes: guidelines to chase them off. Med Vet Entomol 20: 157-165.

Alonso WJ, Wyatt TD, Kelly DW 2003. Are vectors able to learn about their hosts? A case study with Aedes aegypti mosquitoes. Mem Inst Oswaldo Cruz 98: 665-672.

Alzogaray RA, Fontan A, Camps F, Masuh H, Santo OP, Fernandez D, Cork A, Zerba E 2005. Behavioural response of Triatoma infestans (Klug) (Hemiptera: Reduviidae) to quinazolines. Molecules 10: 1190-1196.

Ampleford EJ, Steel CGH 1982. Circadian control of ecdysis in Rhodnius prolixus (Hemiptera). J Comp Physiol A 147: 281-286.

Baldwin WF, Knight AG, Lyn KR 1971. A sex pheromone in the insect Rhodnius prolixus (Hemiptera: Reduviidae). Can Entomol 103: $18-22$.

Barbu C, Dumonteil E, Gourbiere S 2009. Optimization of control strategies for non-domiciliated Triatoma dimidiata, Chagas disease vector in the Yucatan Peninsula, Mexico. PLoS Negl Trop Dis 3: e416.
Barbu C, Dumonteil E, Gourbiere S 2010. Characterization of the dispersal of non-domiciliated Triatoma dimidiata through the selection of spatially explicit models. PLoS Negl Trop Dis 4: e777.

Barrett FM, Millen BF, Laifook J 1979. Brindleys Glands of Rhodnius prolixus. 1. Structure of the mature gland. Can J Zool 57: 1109-1119.

Barrozo RB, Lazzari CR 2004a. The response of the blood-sucking bug Triatoma infestans to carbon dioxide and other host odours. Chem Senses 29: 319-329.

Barrozo RB, Lazzari CR 2004b. Orientation behaviour of the bloodsucking bug Triatoma infestans to short-chain fatty acids: synergistic effect of L-lactic acid and carbon dioxide. Chem Senses 29: 833-841.

Barrozo RB, Manrique G, Lazzari CR 2003. The role of water vapour in the orientation behaviour of the blood-sucking bug Triatoma infestans (Hemiptera, Reduviidae). J Insect Physiol 49: 315-321.

Barrozo RB, Minoli SA, Lazzari CR 2004a. Circadian rhythm of behavioural responsiveness to carbon dioxide in the blood-sucking bug Triatoma infestans (Heteroptera: Reduviidae). J Insect Physiol 50: 249-254.

Barrozo RB, Schilman PE, Minoli SA, Lazzari CR 2004b. Daily rhythms in disease-vector insects. Biol Rhythm Res 35: 79-92.

Bennet-Clark HC 1963. Negative pressures produced in the pharyngeal pump of the blood-tucking bug, Rhodnius prolixus. $J$ Exp Biol 40: 223-229.

Bernier UR, Kline DL, Barnard DR, Schreck CE, Yost RA 2000. Analysis of human skin emanations by gas chromatography/mass spectrometry. 2. Identification of volatile compounds that are candidate attractants for the yellow fever mosquito (Aedes aegypti). Anal Chem 72: 747-756.

Bodin A, Barrozo RB, Couton L, Lazzari CR 2008. Temporal modulation and adaptive control of the behavioural response to odours in Rhodnius prolixus. J Insect Physiol 54: 1343-1348.

Bodin A, Vinauger C, Lazzari CR 2009a. Behavioural and physiological state dependency of host seeking in the bloodsucking insect Rhodnius prolixus. J Exp Biol 212: 2386-2393.

Bodin A, Vinauger C, Lazzari CR 2009b. State-dependency of hostseeking in Rhodnius prolixus: the post-ecdysis time. J Insect Physiol 55: 574-579.

Bosch OJ, Geier M, Boeckh J 2000. Contribution of fatty acids to olfactory host finding of female Aedes aegypti. Chem Senses 25: 323-330.

Botto-Mahan C, Cattan PE, Medel R 2006. Chagas disease parasite induces behavioural changes in the kissing bug Mepraia spinolai. Acta Trop 98: 219-223.

Carbajal de la Fuente AL, Minoli SA, Lopes CM, Noireau F, Lazzari CR, Lorenzo MG 2007. Flight dispersal of the Chagas disease vectors Triatoma brasiliensis and Triatoma pseudomaculata in northeastern Brazil. Acta Trop 101: 115-119.

Clayton J 2010. Chagas disease 101. Nature 465: S4-S5.

Cocchiararo-Bastias LM, Mijailovsky SJ, Calderon-Fernández GM, Lorenzo Figueiras AN, Juarez P 2011. Epicuticle lipids mediate mate recognition in Triatoma infestans. J Chem Ecol 37: 246-252.

Constantinou C 1984. Circadian rhythm of oviposition in the blood sucking bugs, Triatoma phyllosoma, Triatoma infestans and Panstrongylus megistus (Hemiptera, Reduviidae). J Interdiscipl Cycle Res 15: 203-211.

Cork A, Park KC 1996. Identification of electrophysiologically-active compounds for the malaria mosquito, Anopheles gambiae, in human sweat extracts. Med Vet Entomol 10: 269-276. 
Coura JR, Borges-Pereira J 2007. Chagas disease: 100 years after its discovery. A systemic review. Acta Trop 115: 5-13.

Crespo JG, Manrique G 2007. Mating behavior of the hematophagous bug Triatoma infestans: role of Brindley's and metasternal glands. J Insect Physiol 53: 708-714.

Cruz-Lopez L, Malo EA, Rojas JC, Morgan ED 2001. Chemical ecology of triatomine bugs: vectors of Chagas disease. Med Vet Entomol 15: 351-357.

Cruz-Lopez L, Morgan ED, Ondarza RN 1995. Brindley's gland exocrine products of Triatoma infestans. Med Vet Entomol 9: 403- 406.

Dias JC, Silveira AC, Schofield CJ 2002. The impact of Chagas disease control in Latin America: a review. Mem Inst Oswaldo Cruz 97: 603-612.

Dumonteil E, Gourbiere S, Barrera-Perez M, Rodriguez-Felix E, Ruiz-Pina H, Banos-Lopez O, Ramirez-Sierra MJ, Menu F, Rabinovich JE 2002. Geographic distribution of Triatoma dimidiata and transmission dynamics of Trypanosoma cruzi in the Yucatan peninsula of Mexico. Am J Trop Med Hyg 67: 176-183.

Ferreira RA, Lazzari CR, Lorenzo MG, Pereira MH 2007. Do haematophagous bugs assess skin surface temperature to detect blood vessels? PLOS ONE 2: e932.

Figueiras ANL, Lazzari CR 1998a. Aggregation behaviour and interspecific responses in three species of Triatominae. Mem Inst Oswaldo Cruz 93: 133-137.

Figueiras ANL, Lazzari CR 1998b. Aggregation in the haematophagous bug Triatoma infestans: a novel assembling factor. Physiol Entomol 23: 33-37.

Figueiras ANL, Lazzari CR 2000. Temporal change of the aggregation response in Triatoma infestans. Mem Inst Oswaldo Cruz 95: 889-892.

Fontan A, Audino PG, Martinez A, Alzogaray RA, Zerb EN, Camps F, Cork A 2002. Attractant volatiles released by female and male Triatoma infestans (Hemiptera: Reduviidae), a vector of Chagas disease: chemical analysis and behavioral bioassay. J Med Entomol 39: 191-197.

Fresquet N, Lazzari CR 2011. Response to heat in Rhodnius prolixus: the role of the thermal background. J Insect Physiol 57: 1446-1449.

Friend WG, Smith JJ 1977. Factors affecting feeding by bloodsucking insects. Annu Rev Entomol 22: 309-331.

Garrouste R 2009. First observation in natura of entomophagy of Panstrongylus geniculatus (Latreille 1811), vector of Chagas disease (Hemiptera: Reduviidae). Ann Soc Entomol France 45: 302-304.

Giurfa M 2003. Cognitive neuroethology: dissecting non-elemental learning in a honeybee brain. Curr Opin Neurobiol 13: 726-735.

Giurfa M 2004. Comportement et cognition: ce que nous apprend un mini-cerveau. In M Kreutzer, J Vauclair (eds.), L'ethologie cognitive, Orphys, Maison des Sciences de l'Homme, Paris, p. 83-99.

Giurfa M, Zhang S, Jenett A, Menzel R, Srinivasan MV 2001. The concepts of 'sameness' and 'difference' in an insect. Nature 410: $930-933$.

Gomez Núñez JC 1965. Desarrollo de un nuevo método para evaluar la infestación intradomiciliaria por Rhodnius prolixus. Acta Cient Venez 16: 26-31.

Guarneri AA, Lazzari C, Diotaiuti L, Lorenzo MG 2002. The effect of relative humidity on the behaviour and development of Triatoma brasiliensis. Physiol Entomol 27: 142-147.

Guarneri AA, Lazzari C, Xavier AAP, Diotaiuti L, Lorenzo MG 2003. The effect of temperature on the behaviour and development of Triatoma brasiliensis. Physiol Entomol 28: 185-191.
Guerenstein PG, Guerin PM 2001. Olfactory and behavioural responses of the blood-sucking bug Triatoma infestans to odours of vertebrate hosts. $J$ Exp Biol 204: 585-597.

Guerenstein PG, Guerin PM 2004. A comparison of volatiles emitted by adults of three triatomine species. Entomol Exp Appl 111: 151-155.

Guerenstein PG, Hildebrand JG 2008. Roles and effects of environmental carbon dioxide in insect life. Annu Rev Entomol 53: 161-178.

Guerenstein PG, Lazzari CR 2009. Host-seeking: how triatomines acquire and make use of information to find blood. Acta Trop 110: $148-158$.

Guerenstein PG, Lorenzo MG, Núñez JA, Lazzari CR 1995. Baker's yeast, an attractant for baiting traps for Chagas' disease vectors. Experientia 51: 834-837.

Guerenstein PG, Núñez JA 1994. Feeding response of the hematophagous bugs Rhodnius prolixus and Triatoma infestans to saline solutions. A comparative-study. J Insect Physiol 40: 747-752.

Gurevitz JM, Ceballos LA, Kitron U, Gurtler RE 2006. Flight initiation of Triatoma infestans (Hemiptera: Reduviidae) under natural climatic conditions. J Med Entomol 43: 143-150.

Jander R 1963. Insect orientation. Annu Rev Entomol 8: 95-114.

Kelly DW 2001. Why are some people bitten more than others? Trends Parasitol 17: 578-581.

Kelly DW, Thompson CE 2000. Epidemiology and optimal foraging: modelling the ideal free distribution of insect vectors. Parasitology 120: 319-327.

Kingsolver JG, Daniel TL 1995. Mechanics of food handling by 42 fluid-feeding insects. In RFChapman, G de Boer (eds.), Regulatory mechanisms in insect feeding, Chapman \& Hall, New York, p. 32-73.

Lazzari CR 1991a. Circadian rhythm of egg hatching in Triatoma infestans (Hemiptera, Reduviidae). J Med Entomol 28: 740-741.

Lazzari CR 1991b. Temperature preference in Triatoma infestans (Hemiptera, Reduviidae). Bull Entomol Res 81: 273-276.

Lazzari CR 1992. Circadian organization of locomotion activity in the hematophagous bug Triatoma infestans. J Insect Physiol 38: 895-903.

Lazzari CR 2009. Orientation towards hosts in haematophagous insects: an integrative perspective. Adv Insect Physiol 37: 1-58.

Lazzari CR, Fischbein D, Insausti, TC 2011. Differential control of light-dark adaptation in the ocelli and compound eyes of Triatoma infestans. J Insect Physiol 57: 1545-1550.

Lazzari CR, Manrique G, Schilman PE 2006. Vibrational communication in Triatominae (Heteroptera: Reduviidae). In Insect sounds and communication. Physiology, behaviour, ecology and evolution, JA Drumond, M Claridge (eds.), CRC Press, Boca Raton, p. 297-304.

Lazzari CR, Minoli SA, Barrozo RB 2004. Chemical ecology of insect vectors: the neglected temporal dimension. Trends Parasitol 20: $506-507$

Lazzari CR, Núñez JA 1989a. Blood temperature and feeding behavior in Triatoma infestans (Heteroptera, Reduviidae). Entomol Gener 14: 183-188.

Lazzari CR, Núñez JA 1989b. The response to radiant heat and the estimation of the temperature of distant sources in Triatoma infestans. J Insect Physiol 35: 525-529.

Lazzari CR, Reiseman CE, Insausti TC 1998. The role of the ocelli in the phototactic behaviour of the haematophagous bug Triatoma infestans. J Insect Physiol 44: 1159-1162. 
Lehane MJ 2005. The biology of blood-sucking in insects, Cambridge University Press, New York, 321 pp.

Lent H, Wygodzinsky P 1979. Revision of the Triatominae (Hemiptera, Reduviidae) and their signficance as vectors of Chagas' disease. Bull Am Mus Nat Hist 163: 123-520.

Lorenzo Figueiras AN, Girotti JR, Mijailovsky SJ, Juarez MP 2009. Epicuticular lipids induce aggregation in Chagas disease vectors. Parasit Vectors 2: 8 .

Lorenzo Figueiras AN, Kenigsten A, Lazzari CR 1994. Aggregation in the hematophagous bug Triatoma infestans - Chemical signals and temporal pattern. J Insect Physiol 40: 311-316.

Lorenzo MG, Flores GB, Lazzari CR, Reisenman CE 1999a. Sensory ecology. A. Orientation. In RU Carcavallo, I Galindez-Giron, J Jurberg, H Lent (eds.), Atlas of Chagas' disease vectors in America, Vol. III, Fiocruz, Rio de Janeiro, p. 1071-1087.

Lorenzo MG, Lazzari CR 1996. The spatial pattern of defaecation in Triatoma infestans and the role of faeces as a chemical mark of the refuge. J Insect Physiol 42: 903-907.

Lorenzo MG, Lazzari CR 1998. Activity pattern in relation to refuge exploitation and feeding in Triatoma infestans (Hemiptera : Reduviidae). Acta Trop 70: 163-170.

Lorenzo MG, Lazzari CR 1999. Temperature and relative humidity affect the selection of shelters by Triatoma infestans, vector of Chagas disease. Acta Trop 72: 241-249.

Lorenzo MG, Manrique G, Pires HH, de Brito Sanchez MG, Diotaiuti L, Lazzari CR 1999b. Yeast culture volatiles as attractants for Rhodnius prolixus: electroantennogram responses and captures in yeast-baited traps. Acta Trop 72: 119-124.

Lorenzo MG, Reisenman CE, Lazzari CR 1998. Triatoma infestans can be captured under natural climatic conditions using yeastbaited traps. Acta Trop 70: 277-284.

Lorosa ES, Jurberg J, Souza ALA, Vinhaes MC, Nunes IM 2000. Haemolymph of Dictyoptera in the maintenance of the biological cycle of wild Triatoma rubrovaria (Blanchard, 1843) and Triatoma circummaculata (Stål, 1859) (Hemiptera, Reduviidae, Triatominae). Entomología y Vectores 7 : 287-295.

Manrique G, Lazzari CR 1994. Sexual behaviour and stridulation during mating in Triatoma infestans (Hemiptera: Reduviidae). Mem Inst Oswaldo Cruz 89: 629-633.

Manrique G, Lazzari CR 1995. Existence of a sex pheromone in Triatoma infestans (Hemiptera: Reduviidae): I. Behavioural evidence. Mem Inst Oswaldo Cruz 90: 645-648.

Manrique G, Schilman PE 2000. Two different vibratory signals in Rhodnius prolixus (Hemiptera: Reduviidae). Acta Trop 77: 271-278.

Manrique G, Vitta AC, Ferreira RA, Zani CL, Unelius CR, Lazzari CR, Diotaiuti L, Lorenzo MG 2006. Chemical communication in Chagas disease vectors. Source, identity and potential function of volatiles released by the metasternal and Brindley's glands of Triatoma infestans adults. J Chem Ecol 32: 2035-2052.

May-Concha I, Rojas JC, Cruz-Lopez L, Millar JG, Ramsey JM 2013. Volatile compounds emitted by Triatoma dimidiata, a vector of Chagas disease: chemical analysis and behavioural evaluation. Med Vet Entomol 27: 165-174.

McCall PJ, Kelly DW 2002. Learning and memory in disease vectors. Trends Parasitol 18: 429-433.

Minoli SA, Lazzari CR 2003. Chronobiological basis of thermopreference in the haematophagous bug Triatoma infestans. J Insect Physiol 49: 927-932.

Minoli SA, Lazzari CR 2006. Take-off activity and orientation of triatomines (Heteroptera: Reduviidae) in relation to the presence of artificial lights. Acta Trop 97: 324-330.
Minoli SA, Palotini F, Manrique G 2013. The main component of an alarm pheromone of kissing bugs plays multiple roles in the cognitive modulation of the escape response. Front Behav Neurosci 7: 77 .

Noireau F, Abad-Franch F, Valente SAs, Dias-Lima A, Lopes CM, Cunha V, Valente VC, Palomeque FS, de Carvalho-Pinto CJ, Sherlock I, Aguilar M, Steindel M, Grisard EC, Jurberg J 2002. Trapping Triatominae in silvatic habitats. Mem Inst Oswaldo Cruz 97: 61-63.

Noireau F, Dujardin JP 2001. Flight and nutritional status of sylvatic Triatoma sordida and Triatoma guasayana. Mem Inst Oswaldo Cruz 96: 385-389.

Noireau F, Flores R, Vargas F 1999. Trapping sylvatic Triatominae (Reduviidae) in hollow trees. Trans R Soc Trop Med Hyg 93: 13-14.

Núñez JA 1982. Food source orientation and activity in Rhodnius prolixus Stål (Hemiptera, Reduviidae). Bull Entomol Res 72: 253-262.

Ortiz MI, Molina J 2010. Preliminary evidence of Rhodnius prolixus (Hemiptera: Triatominae) attraction to human skin odour extracts. Acta Trop 113: 174-179.

Pacheco-Tucuch FS, Ramirez-Sierra MJ, Gourbiere S, Dumonteil E 2012. Public street lights increase house infestation by the Chagas disease vector Triatoma dimidiata. PLoS ONE 7: e36207.

Pedrini N, Mijailovsky SJ, Girotti JR, Stariolo R, Cardozo RM, Gentile A, Juarez MP 2009. Control of pyrethroid-resistant Chagas disease vectors with entomopathogenic fungi. PLoS Negl Trop Dis 3: e434.

Pereira MH, Gontijo NF, Guarneri AA, Sant'anna MR, Diotaiuti L 2006. Competitive displacement in Triatominae: the Triatoma infestans success. Trends Parasitol 22: 516-520.

Pires HH, Lazzari CR, Diotaiuti L, Lorenzo MG 2000. Performance of yeast-baited traps with Triatoma sordida, Triatoma brasiliensis, Triatoma pseudomaculata and Panstrongylus megistus in laboratory assays. Rev Panam Salud Publica 7: 384-388.

Pires HH, Lazzari CR, Schilman PE, Diotaiuti L, Lorenzo MG 2002a. Dynamics of thermopreference in the Chagas disease vector Panstrongylus megistus (Hemiptera: Reduviidae). J Med Entomol 39: 716-719.

Pires HH, Lorenzo MG, Diotaiuti L, Lazzari CR, Lorenzo Figueiras AN 2002b. Aggregation behaviour in Panstrongylus megistus and Triatoma infestans: inter and intraspecific responses. Acta Trop 81: 47-52.

Pontes GB 2010. Comportamento sexual de Rhodnius prolixus, $\mathrm{PhD}$ Thesis, Fiocruz, Belo Horizonte, 160 pp.

Pontes GB, Bohman B, Unelius CR, Lorenzo MG 2008. Metasternal gland volatiles and sexual communication in the triatomine bug, Rhodnius prolixus. J Chem Ecol 34: 450-457.

Pontes GB, Noireau F, Lorenzo MG 2011. Behavioral evidence of an ectoparasitic interaction between Triatoma pseudomaculata Corrêa e Espínola (Heteroptera: Reduviidae) and Periplaneta americana (L.) (Blattodea: Blattidae). Neotrop Entomol 240: 708-710.

Readio PA 1927. Studies on the biology of Reduviidae of America. North of Mexico. Kansas Univ Sc Bull 17: 5-291.

Reisenman CE, Figueiras ANL, Giurfa M, Lazzari CR 2000. Interaction of visual and olfactory cues in the aggregation behaviour of the haematophagous bug Triatoma infestans. J Comp Physiol A 186: 961-968.

Reisenman CE, Insausti TC, Lazzari CR 2002. Light-induced and circadian changes in the compound eye of the haematophagous bug Triatoma infestans (Hemiptera: Reduviidae). J Exp Biol 205: 201-210. 
Reisenman CE, Lazzari C 2006. Spectral sensitivity of the photonegative reaction of the blood-sucking bug Triatoma infestans (Heteroptera: Reduviidae). J Comp Physiol A 192: 39-44.

Reisenman CE, Lazzari CR, Giurfa M 1998. Circadian control of photonegative sensitivity in the haematophagous bug Triatoma infestans. J Comp Physiol A 183: 533-541.

Reisenman CE, Lee Y, Gregory T, Guerenstein PG 2013. Effects of starvation on the olfactory responses of the blood-sucking bug Rhodnius prolixus. J Insect Physiol 59: 717-721.

Rich C, Longcore T 2006. Ecological consequences of artificial night lighting, Island Press, Washington, $458 \mathrm{pp}$.

Roca MJ, Lazzari CR 1994. Effects of relative humidity on the hematophagous bug Triatoma infestans: hygropreference and eclosion success. J Insect Physiol 40: 901-907.

Roces F, Manrique G 1996. Different stridulatory vibrations during sexual behaviour and disturbance in the blood-sucking bug Triatoma infestans (Hemiptera: Reduviidae). J Insect Physiol 42: 231-238.

Rojas de Arias A, Abad-Franch F, Acosta N, López E, González N, Zerba E, Tarelli G, Masuh H 2012. Post-control surveillance of Triatoma infestans and Triatoma sordida with chemically-baited sticky traps. PLoS Negl Trop Dis 6: e1822.

Ryelandt J, Noireau F, Lazzari CR 2011. A multimodal bait for trapping blood-sucking arthropods. Acta Trop 117: 131-136.

Schaub GA 2006. Parasitogenic alterations of vector behaviour. Int $J$ Med Microbiol 296 (Suppl.): 37-40.

Schilman PE, Lazzari CR 2004. Temperature preference in Rhodnius prolixus, effects and possible consequences. Acta Trop 90: $115-122$.

Schilman PE, Lazzari CR, Manrique G 2001. Comparison of disturbance stridulations in five species of triatominae bugs. Acta Trop 79: $171-178$.

Schilman PE, Minoli SA, Lazzari CR 2009. The adaptive value of hatching towards the end of the night: lessons from eggs of the haematophagous bug Rhodnius prolixus. Physiol Entomol 34: 231-237.

Schmitz H, Trenner S, Hofmann MH, Bleckmann H 2000. The ability of Rhodnius prolixus (Hemiptera: Reduviidae) to approach a thermal source solely by its infrared radiation. J Insect Physiol 46: $745-751$.

Schofield CJ 1977. Sound production in some triatominae bugs. Physiol Entomol 2: 43-52.

Schofield CJ, Patterson JW 1977. Assembly pheromone of Triatoma infestans and Rhodnius prolixus nymphs (Hemiptera: Reduviidae). J Med Entomol 13: 727-734.

Smallegange RC, Schmied WH, van Roey KJ, Verhulst NO, Spitzen J, Mukabana WR, Takken W 2010. Sugar-fermenting yeast as an organic source of carbon dioxide to attract the malaria mosquito Anopheles gambiae. Malaria J 9: 292.

Steel CG, Vafopoulou X 2006. Circadian orchestration of developmental hormones in the insect, Rhodnius prolixus. Comp Biochem Physiol A Mol Integr Physiol 144: 351-364.

Takano-Lee M, Edman JD 2002. Lack of manipulation of Rhodnius prolixus (Hemiptera: Reduviidae) vector competence by Trypanosoma cruzi. J Med Entomol 39: 44-51.

Taneja J, Guerin PM 1995. Oriented responses of the triatomine bugs Rhodnius prolixus and Triatoma infestans to vertebrate odors on a servosphere. J Comp Physiol A 176: 455-464.
Taneja J, Guerin PM 1997. Ammonia attracts the haematophagous bug Triatoma infestans: behavioural and neurophysiological data on nymphs. J Comp Physiol A 181: 21-34.

Vafopoulou X, Steel CG, Terry KL 2007. Neuroanatomical relations of prothoracicotropic hormone neurons with the circadian timekeeping system in the brain of larval and adult Rhodnius prolixus (Hemiptera). J Comp Neurol 503: 511-524.

Vafopoulou X, Terry KL, Steel CGH 2010. The circadian timing system in the brain of the fifth larval instar of Rhodnius prolixus (Hemiptera). J Comp Neurol 518: 1264-1282.

Vallejo GA, Guhl F, Schaub GA 2008. Triatominae-Trypanosoma cruzi/T. rangeli: vector-parasite interactions. Acta Trop 110: 137-147.

Vazquez-Prokopec GM, Ceballos LA, Kitron U, Gurtler RE 2004. Active dispersal of natural populations of Triatoma infestans (Hemiptera: Reduviidae) in rural northwestern Argentina. J Med Entomol 41: 614-621.

Vazquez-Prokopec GM, Ceballos LA, Marcet PL, Cecere MC, Cardinal MV, Kitron U, Gurtler RE 2006. Seasonal variations in active dispersal of natural populations of Triatoma infestans in rural north-western Argentina. Med Vet Entomol 20: 273-279.

Vazquez-Prokopec GM, Ceballos LA, Salomón OD, Gürtler RE 2002. Field trials of an improved cost-effective device for detecting peridomestic populations of Triatoma infestans (Hemiptera: Reduviidae) in rural Argentina. Mem Inst Oswaldo Cruz 97: 971-977.

Vinauger C, Buratti L, Lazzari CR 2011a. Learning the way to blood: first evidence of dual olfactory conditioning in a blood-sucking insect, Rhodnius prolixus. Part I: appetitive learning. J Exp Biol 214: 3032-3038.

Vinauger C, Buratti L, Lazzari CR CR 2011b. Learning the way to blood: first evidence of dual olfactory conditioning in a bloodsucking insect, Rhodnius prolixus. Part II: aversive learning. J Exp Biol 214: 3039-3045.

Vinauger C, Lallement H, Lazzari CR 2013. Learning and memory in Rhodnius prolixus: habituation and aversive operant conditioning of the proboscis extension response (PER). J Exp Biol 216: 892-900.

Vinauger C, Pereira MH, Lazzari CR 2012. Learned host preference in a Chagas disease vector, Rhodnius prolixus. Acta Trop. 122: 24-28.

Vitta ACR, Lorenzo MG 2009. Copulation and mate guarding behaviour in Triatoma brasiliensis. J Med Entomol 46: 789-795.

Vitta ACR, Mota TRP, Diotaiuti L, Lorenzo MG 2007. The use of aggregation signals by Triatoma brasiliensis (Heteroptera: Reduviidae). Acta Tropica 101: 147-152.

Ward JP 1981. A comparison of the behavioral responses of the hematophagous bug, Triatoma infestans, to synthetic homologs of two naturally-occurring chemicals (N-butyric and iso-butyric acid). Physiol Entomol 6: 325-329.

Wigglesworth VB, Gillet JD 1934. The function of the antennae in Rhodnius prolixus (Hemiptera) and the mechanism of orientation to the host. J Exp Biol 11: 120-138.

Wisnivesky-Colli C, Paulone I, Perez A, Chuit R, Gualtieri J, Solarz N, Smith A, Segura EL 1987. A new tool for continuous detection of the presence of triatomine bugs, vectors of Chagas disease, in rural households. Medicina (B Aires) 47: 45-50.

Zacharias CA, Pontes GB, Lorenzo MG, Manrique G 2010. Flight initiation by male Rhodnius prolixus is promoted by female odors. J Chem Ecol 36: 449-451. 